Ann. Biol. anim. Bioch. Biophys., 1972, 12 (3), 367-375.

\title{
APPLICATION D'UN SYSTEME SEMESTRIEL DE VARIATION DE LA PHOTOPERIODE CHEZ MICROCEBUS MURINUS (MILLER, 1777)
}

\author{
Arlette PETTER-ROUSSEAUX \\ Laboratoire d'Écologie, \\ Muséum d'Histoire naturelle, \\ 91800 Brunoy
}

\section{RÉSUMÉ,}

Les Microcèbes, soumis à un régime de variations semestrielles de la photopériode présentent deux périodes d'activité sexuelle par année.

Chaque période correspond, chez le mâle, au développement testiculaire, et chez la femelle à l'apparition d'un cestrus. Les photopériodes croissantes induisent l'activité et les photopériodes décroissantes provoquent une mise au repos précoce. Une variation de poids accompagne l'activité sexuelle, le poids minimum étant atteint après la période d'activité sexuelle, et le poids maximum après la période de repos.

\section{INTRODUCTION}

Nous avons montré, dans un précédent travail, que la période annuelle d'activité sexuelle, chez Microcebus murinus, dépend du photopériodisme : elle a lieu en jours croissants, et elle apparaît à contre-saison sous l'action du changement d'hémisphère ou d'un éclairement de jours longs appliqué pendant trois mois environ.

En nous basant sur ces résultats, nous avons cherché à savoir dans quelle mesure il était possible d'instaurer, chez ces animaux en élevage, une succession de deux périodes d'activité sexuelle par année.

\section{MATÉRIEL ET MÉTHODES}

Nos animaux sont installés par 2 ou 3 dans des cages de $50 \mathrm{~cm}$ de côté, où ils logent dans de petites niches en bois. Ils sont nourris de fruits, lait, vers de farine, miel, viande crue. La température de l'animalerie est d'environ $25^{\circ} \mathrm{C}$. 
Pendant la durée de l'expérience, nous avons pesé les animaux au moins tous les deux mois, et nous avons mesuré, extérieurement, au pied à coulisse, la largeur et la longueur des testicules, environ toutes les trois semaines.

Cette dernière mesure n'est évidemment pas très précise, mais la variation est telle, de la saison de repos à la saison d'activité, que l'erreur commise ( $2 \mathrm{~mm}$ environ) est relativement peu importante : la longueur du testicule est de 19 à $22 \mathrm{~mm}$ suivant les individus, en saison d'activité. Le testicule régresse peu à peu en jours décroissants, et la mesure est alors de $8 \mathrm{~mm}$ environ. A partir de ce moment, il remonte légèrement vers le canal inguinal, et les replis de peau et de graisse empêchent de le mesurer correctement. Il reste dans cette position pendant la saison de repos; les mesures faites à la dissection sont alors d'environ $5 \mathrm{~mm}$ de longueur.

Chaque femelle a été examinée deux fois par semaine pendant la période d'activité pour noter l'apparition de l'œstrus vaginal qui se reconnaît extérieurement au gonflement, au rougissement et à l'ouverture de la vulve.

Nos animaux ont été répartis dans trois pièces, dans les conditions suivantes :

Io $5 \delta^{x}$ et I 2 ㅇ dans une pièce en éclairement naturel; ces animaux étaient depuis au moins deux années sous éclairement naturel.

$2^{\circ} 5 \delta$ et 15 dans une pièce éclairée artificiellement, avec variation de longueur des jours imitant le rythme lumineux malgache ; les heures d'allumage et d'extinction ont été déterminées d'après le relevé des heures de lever et de coucher đu soleil fait à Tananarive par l'Office météorologique (latitude : $19^{\circ}$ ).

$3^{\circ} 5$ ot et I I 9 dans une pièce éclairée artificiellement selon un rythme semestriel ; l'amplitude des variations adoptée a été celle du rythme malgache.

Les variations de longueur des jours au cours de l'année, ont été portées sur un graphique (fig. I). Dans la pièce en lumière naturelle, les jours les plus longs se situent vers le 20 juin; ce

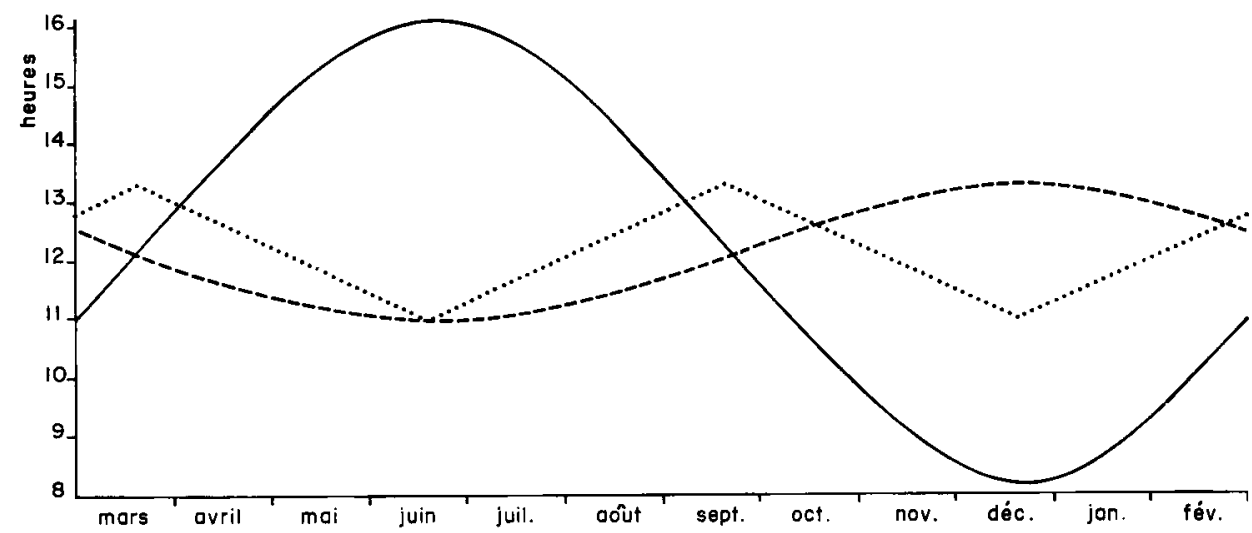

Fig. I. - Variations de longueur des jours au cours de l'année

en trait plein : en eclairement naturel à Paris.

en tirets : en éclairement artificiel imitant le rythme malgache;

en pointillés : en éclairement artificiel de rythme semestriel, et de même amplitude de variation que le rythme malgache.

moment est celui des jours les plus courts dans la pièce à rythme malgache. Dans la pièce à deux printemps, la photopériode augmente ou diminue uniformément pendant trois mois; il y a inversion aux jours les plus longs (20 septembre et 20 mars), et les plus courts (20 juin et 20 décembre). Chacune des deux périodes annuelles de jours croissants accompagne celle d'une des deux autres pièces.

L'allumage et l'extinction se font brutalement. L'augmentation et la diminution de longueur des jours sont obtenus par un réajustement tous les ro jours, ce qui donne une modification de durée de 5 minutes à la fois et au maximum pour le rythme lumineux malgache, et de 5 minutes à la fois et régulièrement pour l'expérience.

Les observations ont été faites d'avril I969 à novembre I970. Les animaux choisis pour 
l'expérience de rythme semestriel étaient, avant le 20 juin 1969, soumis au rythme d'éclairement malgache; ils étaient au début de l'expérience, en jours courts (II h), et I5 mois plus tard, peu

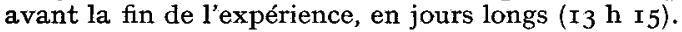

Durée du jour

\begin{tabular}{l|c|c|c}
\hline \multicolumn{1}{c|}{ Date } & $\begin{array}{c}\text { Éclairement } \\
\text { naturel } \\
(\mathrm{h})\end{array}$ & $\begin{array}{c}\text { Rythme lumineux } \\
\text { malgache } \\
(\mathrm{h})\end{array}$ & $\begin{array}{c}\text { Expérience de } \\
\text { rythme semestriel } \\
\text { (h) }\end{array}$ \\
\hline 20 mars ....... & 12,03 & 12,05 & 13,15 \\
20 juin ........ & 16,07 & 11 & 11 \\
20 septembre ... & 12,17 & 12,04 & 13,15 \\
20 décembre ... & 8,12 & 13,15 & 11 \\
\hline
\end{tabular}

Les Microcèbes ont une activité nocturne; pendant le jour, dans les cages, la quantité de lumière reçue varie de 50 à $\mathbf{I} 50$ lux; à l'intérieur des niches où les animaux passent la journée, elle est inférieure à I lux.

\section{RÉSUI,TATS}

\section{I. - Poids des animaux}

En captivité, les Microcèbes subissent des variations annuelles régulières de poids, celui-ci étant minimum à la fin de la saison d'activité sexuelle, et maximum à la fin de la saison de repos.

Les courbes de poids des animaux vivant dans la pièce en lumière naturelle concordent avec les résultats déjà publiés (BOURLIÈRE et PETTER-ROUSSEAUX, I966). Les courbes de poids des animaux éclairés selon le rythme malgache (fig. 2), sont inversées, le poids minimum étant atteint en janvier (jours longs) et le poids maximum entre mai et septembre (jours courts). Dans la pièce expérimentale à rythme semestriel, le poids oscille selon les mêmes règles : poids maximum en février et juillet, après le repos, poids minimum en mai et octobre, après l'activité.

Deux mâles de cette pièce, étant de taille légèrement plus petite (sous-espèce géographique différente), ont été portés séparément sur le graphique ; leur variation de poids suit la courbe générale.

\section{2. 一 Entrées en cestrus}

Toutes les femelles sont entrées en œestrus dans chacune des trois pièces d'élevage.

En lumière naturelle de Paris, II entrées en œestrus, sur un total de 20 , ont eu lieu dans la première quinzaine de mai ( $55 \mathrm{p}$. I00) (fig. 3).

Dans la pièce à rythme malgache, $I 7$ sur 30 entrées en œstrus se sont situées dans la première quinzaine d'octobre $(56 \mathrm{p}$. Ioo). 

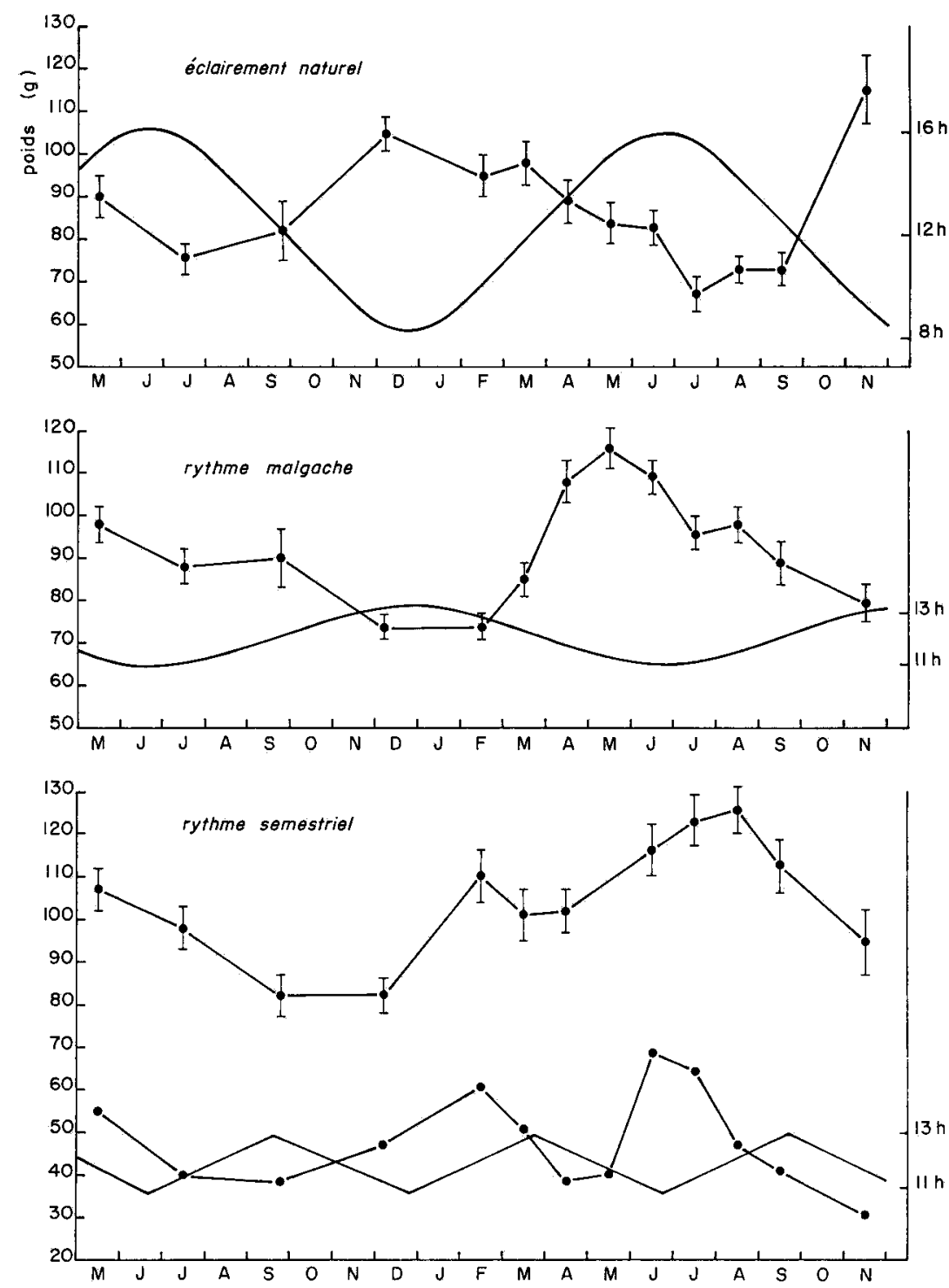

FIG. 2. - Variations annuelles de poids des Microcèbes en éclairement naturel. Moyennes et erreurs-type (haut)

Variations annuelles de poids des Microcèbes éclairés artificiellement selon un rythme malgache. Moyennes et erreurs-type (milieu)

Variations annuelles de poids des Microcèbes éclairés artificiellement selon un rythme semestriel de même amplitude que le rythme malgache

Moyennes et erreurs-type. Le courbe de poids inférieure représente les poids de deux spécimens de taille légèrement inférieure (bas) 
Dans la pièce à rythme semestriel, nous avons obtenu deux saisons d'activité alternant avec deux saisons de repos. L'entrée en œestrus se fait en mars et en septembre (jours longs) ; nous observons une synchronisation plus grande de l'activité sexuelle : 9 I p. Ioo des œstrus (Io sur II), ont lieu dans la seconde quinzaine de mars pour le premier printemps, et $68 \mathrm{p}$. Ioo ( 15 sur 22) dans la seconde quinzaine

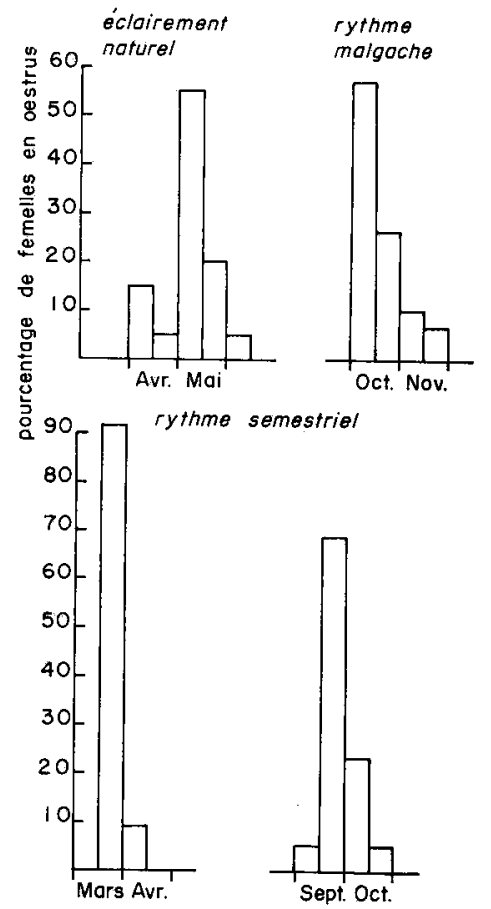

FIG. 3. - Proportions d'entrées en cestrus au cours de chaque quinzaine, pendant la saison d'activité sexuelle

de septembre pour le deuxième printemps. Les entrées en œstrus ont lieu un peu plus tôt après le jour le plus court (d'une quinzaine de jours au moins) chez les animaux en rythme semestriel que chez les animaux en rythme annuel. Enfin, le nombre de cycles œstriens pour une même femelle, qui est de deux à quatre, à une quarantaine de jours d'intervalle, par saison, dans les deux autres pièces, a tendance à se réduire à un dans la pièce expérimentale (deux femelles seulement ayant eu un deuxième ostrus, et seulement lors de la première période d'activité.)

\section{3. - Croissance des testicules (tabl. I)}

En lumière naturelle, les testicules commencent à grossir fin janvier ; le maximum est atteint fin mars ; la diminution s'amorce en juillet et le minimum est atteint fin octobre. Il faut un mois environ pour passer de l'état pleinement actif à l'état de repos. Il peut y avoir $I_{5}$ jours de décalage d'un animal à l'autre. Les jeunes ( $\mathrm{I}$ an $\mathrm{I} / 2$ à 2 ans) ont un développement plus précoce et une activité plus longue. Enfin, les animaux affaiblis ont une régression plus rapide. 
Les animaux éclairés suivant le rythme malgache présentent le même développement saisonnier, mais inversé. Les testicules commencent à grossir fin juillet, sont pleinement développés de septembre à janvier, puis régressent et ne sont plus mesurables fin mars (fig. 4).

TABLEAU I

Longueur des testicules (en $\mathrm{cm}$ )

\begin{tabular}{|c|c|c|c|c|c|c|c|c|c|c|c|c|c|c|c|}
\hline \multirow{2}{*}{ Dates } & \multicolumn{15}{|c|}{ Nombre d'animaux } \\
\hline & 1 & 2 & 3 & 4 & 5 & 1 & 2 & 3 & 4 & 5 & 1 & 2 & 3 & 4 & 5 \\
\hline $8-5-69$ & 1,8 & 1,6 & 1,6 & 1,2 & 2,1 & & & & & & & & & & \\
\hline $24-5$ & 2 & 2 & 1,7 & 1,5 & 2,2 & & & & & & & & & & \\
\hline $6-6$ & 1,8 & 1,8 & 1,7 & 1,5 & 2 & & & & & & & & & & \\
\hline $19-6$ & 1,9 & 1,6 & 1,5 & 1,5 & 1,7 & & & & & & & & & & \\
\hline $2-7$ & 1,9 & 1,5 & 1,5 & 1,3 & 1,9 & & & & & & & & & & \\
\hline $5-8$ & 2,1 & 1,1 & 1,4 & 1,3 & 1,9 & 1,9 & & & 1,4 & & & & & & \\
\hline $21-8$ & 1,4 & 1 & 0,9 & 1,6 & 2,1 & 2 & 1,5 & & 1,9 & 1,8 & & 1,7 & & 1,7 & \\
\hline $9-9$ & 1,2 & 0,8 & 0,8 & 1,5 & 2 & 2 & 1,5 & & 2 & 1,7 & & 1,5 & 1,6 & 1,7 & 1,3 \\
\hline $27-9$ & & & & 1,3 & 1,7 & 2 & 1,5 & 1,3 & 2,2 & 1,7 & 1,7 & 1,6 & 1,8 & 1,9 & 1,7 \\
\hline $18-10$ & & & & & 1,2 & 1,9 & 1,4 & 1,6 & 1,7 & 1,8 & 2,1 & 1,8 & 1,9 & 1,6 & 1,7 \\
\hline $29-11$ & & & & & & 1,8 & 1,8 & 1,7 & 1,6 & 1,6 & 1,6 & 0,9 & 1,5 & & \\
\hline $27-12$ & & & & & & 1,8 & 1,6 & 1,5 & 1,7 & 1,8 & & & & & \\
\hline $11-1-70$ & & & & & 1,4 & 1,7 & 1,3 & 1,5 & & 1,5 & & & & & \\
\hline $31-1$ & 1,6 & & 1,3 & & 2,1 & 1,6 & 1,3 & 1,2 & & & & & & & \\
\hline $14-2$ & 1,7 & & 1,4 & 0,9 & 2 & 1,6 & & & & & & & & & \\
\hline $7-3$ & 1,8 & & 1,4 & 1,6 & 2 & & & & & & 1,3 & & 1,4 & 1,3 & \\
\hline $28-3$ & 1,9 & 1,3 & 1,4 & 1,7 & 2 & & & & & & 2 & 1,3 & 2 & 2 & 2,1 \\
\hline $18-4$ & 1,7 & 1,9 & 1,8 & 1,7 & 2 & & & & & & 1,8 & 1,6 & 1,8 & 2 & 2 \\
\hline $6-5$ & 1,7 & 1,7 & 1,4 & 1,6 & 2 & & & & & & 1,9 & 1,4 & 1,6 & 1,4 & 2 \\
\hline $30-5$ & 2 & 1,5 & 1,3 & 1,4 & 2 & & & & & & & & 1,6 & & \\
\hline $20-6$ & 1,4 & 1,6 & 1,3 & 1,3 & 2 & & & & & & & & 1,4 & & \\
\hline $13-7$ & 1,5 & 1,4 & 1,2 & 1,2 & 2 & & & & & & & & & & \\
\hline 23-7 & 1,6 & 1 & 1 & 1 & 2 & 1,5 & & & 1,4 & & & & & & \\
\hline $12-8$ & 1,5 & & & & 2 & 2,2 & & & 1,9 & & & & & & \\
\hline $31-8$ & 1 & & & & 1,3 & 2 & & 1,1 & 2,1 & & & & & & \\
\hline $12-9$ & & & & & & 2 & & 1,3 & 2,1 & 1 & 1,1 & & & 1,5 & \\
\hline $30-10$ & & & & & & 1,9 & 1,4 & 1,6 & 1,6 & 2 & 1,7 & 1,6 & 1,8 & 2 & 1,6 \\
\hline 21-11 & & & & & & 2 & 1,7 & 1,6 & 1,9 & 2,2 & 1,5 & 1,2 & 1,2 & 1,7 & 1,2 \\
\hline Éclairement & & & Tature & & & & Rythn & ne ma & algach & & & Rythm & le sen & nestri & \\
\hline
\end{tabular}

Chez les animaux soumis au rythme semestriel, il y a deux périodes successives de développement testiculaire, de mars à mai et de septembre à novembre. Le développement testiculaire atteint pendant la période active est le même que chez les autres, mais il dure à peine plus d'un mois (jours longs).

\section{DISCUSSION}

A la suite de ces expériences, nous pouvons faire les remarques suivantes : I ${ }^{\circ}$ Comme on pouvait l'espérer, en éclairement artificiel imitant le photopé- 
riodisme malgache, les animaux en captivité conservent leur rythme d'activité sexuelle d'origine pendant au moins deux ans.

$2^{\circ}$ L'amplitude de la variation annuelle d'éclairement n'a apparemment pas d'incidence sur le déroulement de l'activité sexuelle, puisque celle-ci a les mêmes caractéristiques, que les animaux soient exposés au rythme malgache $(2 \mathrm{~h}$ environ d'amplitude) ou au rythme de Paris ( $8 \mathrm{~h}$ environ). Peu de travaux ont étẻ publiés au sujet de l'influence de l'amplitude des variations sur les rythmes physiologiques

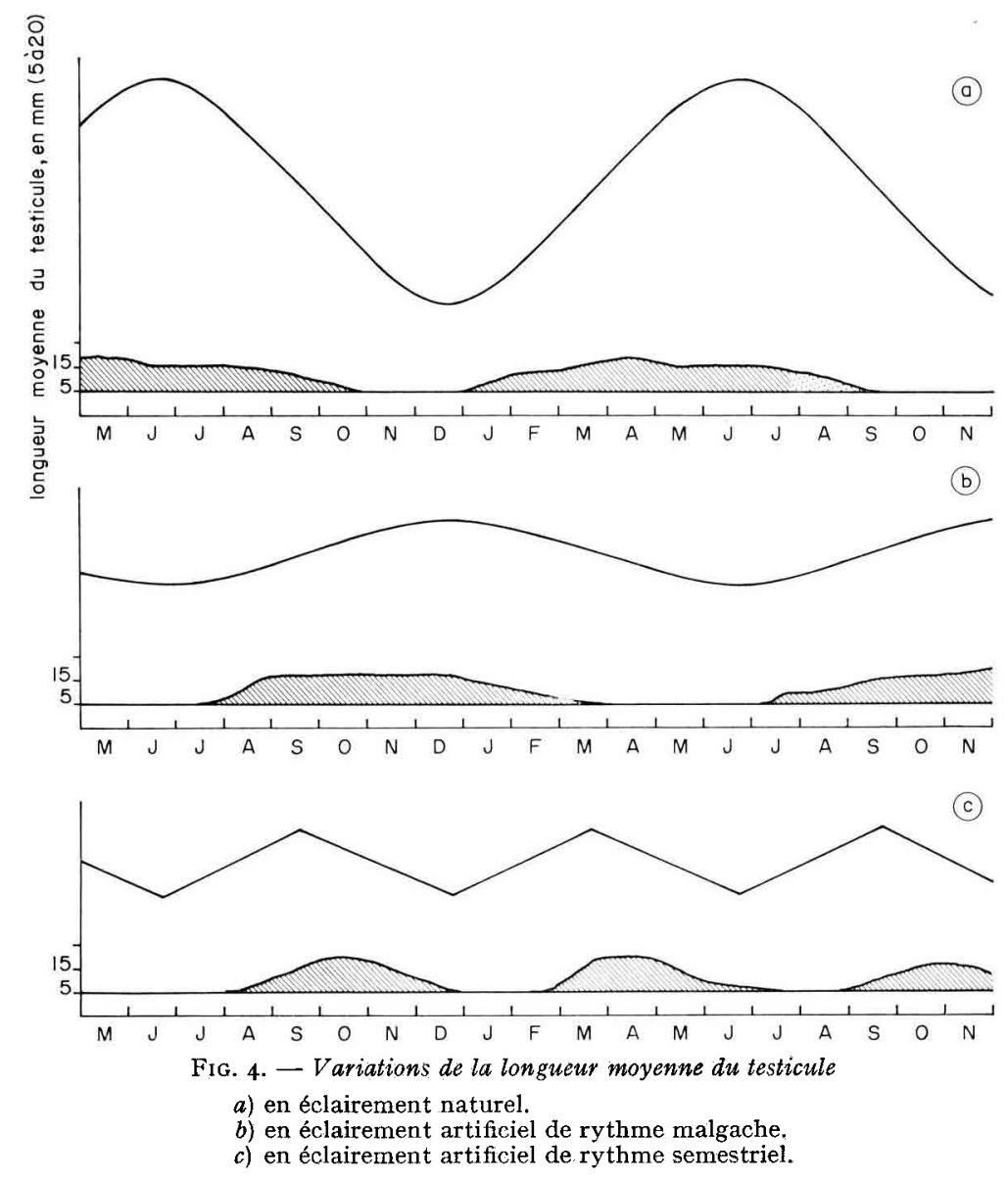

annuels; ils semblent que cetx-ci se conservent sous des amplitudes extrêmement réduites : c'est ainsi que Goss (I969) observe la persistance du phénomène de renouvellement des bois chez le cerf, pour une amplitude de variation photopériodique réduite expérimentalement à I heure.

$3^{\circ}$ Le poids corporel du Microcèbe reflète assez bien les variations d'activité sexuelle, la mise au repos étant suivie d'assez près d'un engraissement de l'animal, et la période active s'accompagnant d'un amaigrissement. Il est intéressant de remarquer que l'augmentation de poids de l'animal est un phénomène d'apparition 
brusque, à l'échelle individuelle et qui peut se réaliser en l'espace d'un mois ; on peut penser que cet engraissement est déclenché par le stimulus des photopériodes décroissantes, comme l'activité sexuelle est déclenchée en jours croissants. Un tel phénomène d'engraissement est d'ailleurs classique chez les Mammifères hibernants des pays tempérés et froids ; il est habituellement opposé à la période d'activité sexuelle. Dans de nombreux cas, il a été démontré qu'il était lié aux photopériodes décroissantes (KAYSER, I967).

$4^{\circ}$ Les Microcèbes répondent parfaitement à l'accélération de rythme de variation de la photopériode, manifestant deux périodes d'activité sexuelle par année dans un rythme semestriel. De tels résultats ont déjà été obtenus chez les brebis ; MAULÉON et ROUGEOT ( $\mathrm{rg62}$ ) observent deux saisons d'activité sexuelle par an, de durée moitié de celle que l'on observe en rythme annuel. Les œestrus, qui ont lieu naturellement en jours courts, apparaissent alors en jours longs; un tel décalage est moins évident chez les Microcèbes, les entrées en oestrus ayant lieu à la fin de la période de jours croissants en expérience, alors qu'elles se situent au milieu de cette période en rythme annuel. Dans le même travail, les auteurs observent que le délai d'apparition des oestrus, à partir du jour le plus long, est plus long en rythme semestriel qu'en rythme annuel ; nous obtenons un résultat semblable pour le grossissement des testicules, qui débute, en rythme annuel, quelquefois moins d'un mois après le jour le plus court, et deux mois seulement après cette date, en rythme semestriel. Les cstrus, au contraire, ont lieu, en moyenne, une quinzaine de jours plus tôt en rythme semestriel qu'en rythme annuel. On pourrait, dans ce dernier cas, invoquer l'existence d'un seuil de photopériode nécessaire à la stimulation, ce seuil étant atteint plus tôt en rythme semestriel qu'en rythme annuel ; mais ceci n'expliquerait pas le fait que les mâles en expérience ont au contraire du retard sur le rythme annuel ; en réalité, tout se passe comme si mâles et femelles se trouvaient stimulés par les jours croissants, à condition d'avoir subi une période de repos : cette période serait située, chez les animaux en rythme annuel, à la fin de la période de jours décroissants ; chez les animaux en rythme semestriel, les mâles et les femelles réagiraient différemment, les mâles ne se trouvant au repos complet qu'aux jours les plus courts, alors que les femelles sont entrées en repos dès la fin du dernier cycle œstrien de la saison, c'est-à-dire peu après les jours les plus longs.

$5^{\circ}$ Comme dans le cas des Brebis, nous constatons un raccourcissement de la période d'activité sexuelle : chez ces Microcèbes soumis à un rythme semestriel, la diminution de photopériode intervient trop tôt pour permettre un deuxième ostrus (que présentent la majeure partie des autres animaux). Chez ces mâles, les testicules ne restent en pleine activité que deux mois environ : chez eux comme chez les femelles, la décroissance des jours provoque une mise au repos précoce.

Cette expérience montre le rôle prépondérant joué par la photopériode dans les activités métaboliques et sexuelle d'un Primate inférieur. 


\section{SUMMARY}

\section{APPLICATION OF SEMESTRIAL PHOTOPERIODISM \\ TO MICROCEBUS MURINUS (MILLER I777)}

When Microcebes are subjected to semestrial variations of photoperiod, they present two periods of sexual activity per year.

In the male, each period corresponds to testicle development, and in the female it corresponds to the appearance of estrus. Increasing photoperiods cause activity, and decreasing photoperiods indice precocious arrest.

Weight variation accompanies sexual activity, minimum weight being reached after the period of sexual activity, and maximum weight after the period of rest.

\section{RÉFÉRENCES BIBLIOGRAPHIQUES}

Bourlitere F., Petrer-Rousseaux A., I966. Existence probable d'un rythme métabolique saisonnier chez les Cheirogaleinae (Lemuroidea). Fol. Primat., 4, no ${ }_{4}^{4}, 249-256$.

Goss R. J., 1969. Photoperiodic control of antler cycles in Deer. II. Alterations in amplitude. $J$. Exp. Zool., 171, 2, 223-234.

KAYSER C., I967. Photopériode, reproduction et hibernation des Mammifères. Dans : La photorégulation de la Reproduction chez les Oiseaux et les Mammifères ". Colloques internationaux du C. N. R. S. Montpellier. 1967. 409-433.

Mauléon P., Rougeot J., r962. Régulation des saisons sexuelles chez des Brebis de races différentes au moyen de divers rythmes lumineux. Ann. Biol. anim. Bioch. Biophys., $2(3), 209-222$.

Petter-Rousseaux A., r962. Recherches sur la Biologie de la reproduction des Primates inférieurs. Mammalia, 26, no $\mathrm{x}$, suppl. 2, $\mathrm{x}-85$.

Petter-Rousseaux A., r97o. Observations sur l'influence de la photopériode sur l'activité sexuelle chez Microcebus murinus (MIller r777) en captivité. Ann. Biol. anim. Bioch. Biophys., 10, 2, 203-208.

Rovgeot J., I96r. Action comparée des variations photopériodiques annuelles et semestrielles de la durée quotidienne de l'éclairement sur les cycles des follicules des jarres courts de la toison des Brebis Limousines. Relations avec leur cycle de reproduction. Ann. Biol. anim. Bioch. Biophys., 1, 385-402. 